\title{
Acute Vestibular Syndrome in Cerebellar Infarction: A Case Report
}

\author{
Diayanti Tenti Lestari ${ }^{1}$, Hanik Badriyah Hidayati ${ }^{2}$ \\ ${ }^{1}$ Department of Neurology, ${ }^{2}$ Department of Neurology, \\ Faculty of Medicine, Airlangga University, Surabaya, Indonesia \\ Corresponding Author: Diayanti Tenti Lestari
}

\begin{abstract}
Introduction: Acute vestibular syndrome (AVS) is characterized by rapid onset of vertigo, nausea and vomiting, and gait unsteadiness in association with head motion intolerance and nystagmus, lasting days to weeks. Although the majority of AVS patients have acute peripheral vestibulopathy, some may also have brainstem or cerebellar strokes. Cerebellar infarctions sometimes only cause vertigo. The Head Impulse Test, skew deviation, and nystagmus testing provide for great sensitivity and specificity in distinguishing between peripheral vestibular impairment and stroke.
\end{abstract}

Case: A 41-year-old male patient suffered from acute-onset vertigo and dizziness about 5 hours before admission, which started when he started doing his morning routine. Patients also feel gait unsteadiness and almost fall to the left side. There was no weakness in extremities, skew face or slurred speech. Patient's neurological status showed the cerebellar examination was positive left dysmetria, left dysdiadochokinesia, the Romberg test open eye fell to the left, normal Head Impulse Test (HIT), with horizontal bidirectional nystagmus and negative skew deviation test. Cerebellum infarction was discovered using computed tomography imaging. After passing through the acute stroke period, patients are offered symptomatic therapy in the form of betahistine, antiplatelet medication, and vestibular rehabilitation planning. On the tenth day after the onset, the patient's symptoms began to improve.

Conclusion: Proper diagnosis of acute vestibular syndrome will guide the necessary tests. The HINTS oculomotor test at the bedside can detect acute vestibular stroke.
Keywords: acute vestibular syndrome, vertigo, cerebellum, HINTS

\section{INTRODUCTION}

Acute vestibular syndrome is characterized by the sudden onset of dizziness/vertigo, nausea and vomiting, and unsteadiness that lasts from days to weeks. This syndrome causes static and dynamic imbalances in the vestibular system on one or both body sides.(1) The occurrence of dizziness/vertigo due to central lesions usually occurs in association with other neurological signs and symptoms, and the diagnosis of vertigo is isolated from lesions of the brainstem and cerebellum. (1) Dizziness/vertigo is the most common symptom of the posterior circulation ischemia. Based on literature, $62 \%$ patients with vertigo due to vertebrobasilar infarction had at least one episode of vertigo and $19 \%$ patients presented vertigo as the initial symptom. (1) Acute vestibular syndrome due to stroke is mostly due to lesions in the posterior inferior cerebellar artery (PICA) region of cerebellum. (2) Cerebellum lesions cause variety of audiovestibular symptoms and nystagmus. (2) Nystagmus is caused by damage to the intercalate nucleus and paramedian tract (PMT) cell group. (2)

Dizziness and vertigo assemble for $4.0 \%$ of visits to the emergency department and associated stroke in $3.2-4.0 \%$ of cases. $(2,3)$ Data show that approximately 15000 to 25000 cases have a morbidity due to misdiagnoses at the time of initial 
admission. (4) Twenty-five percent of patients with vascular risk factors who present to emergency department with isolated severe vertigo, nystagmus, and postural instability, turned out to have cerebellar infarction in the region of the medial posterior inferior cerebellar artery PICA (mPICA) region. (5) Dizziness/ vertigo caused by acute vestibular syndrome have three times higher risk of cerebrovascular causes than dizziness without the syndrome of vestibular acute.
The prevalence of the causes of vertigo/dizziness Cerebrovascular in acute vestibular syndrome group was $10.0 \%$ of strokes and transitory ischemic attack (TIA), this is higher when compared to the occurrence of cerebrovascular causes among vertigo/dizziness patients who are not acute vestibular syndrome as much as $3.6 \%$. (3) This paper is intended to aid in the study of dizziness or sudden vertigo in posterior circulation strokes, particularly cerebellar infarction.

\section{CASE REPORT}

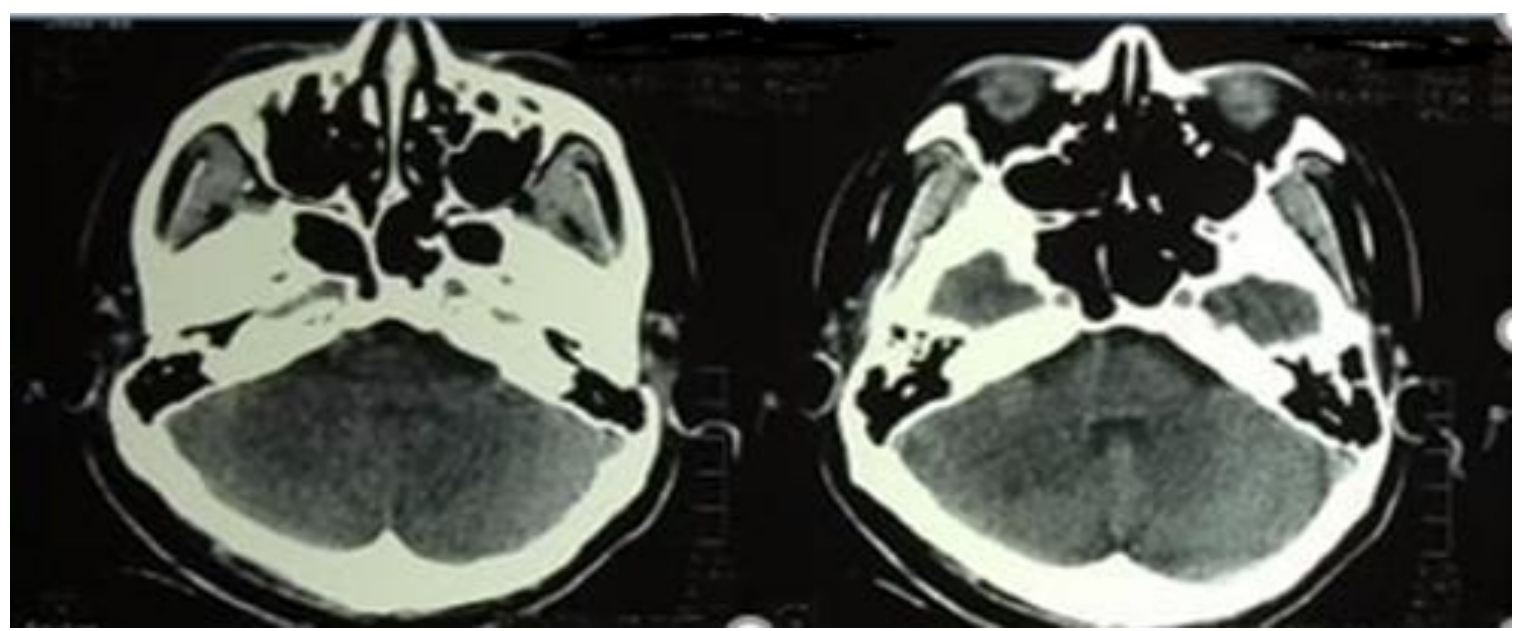

Figure 1. Computed Tomography CT scan of the head without contrast, axial section; acute infarction of the cerebellum

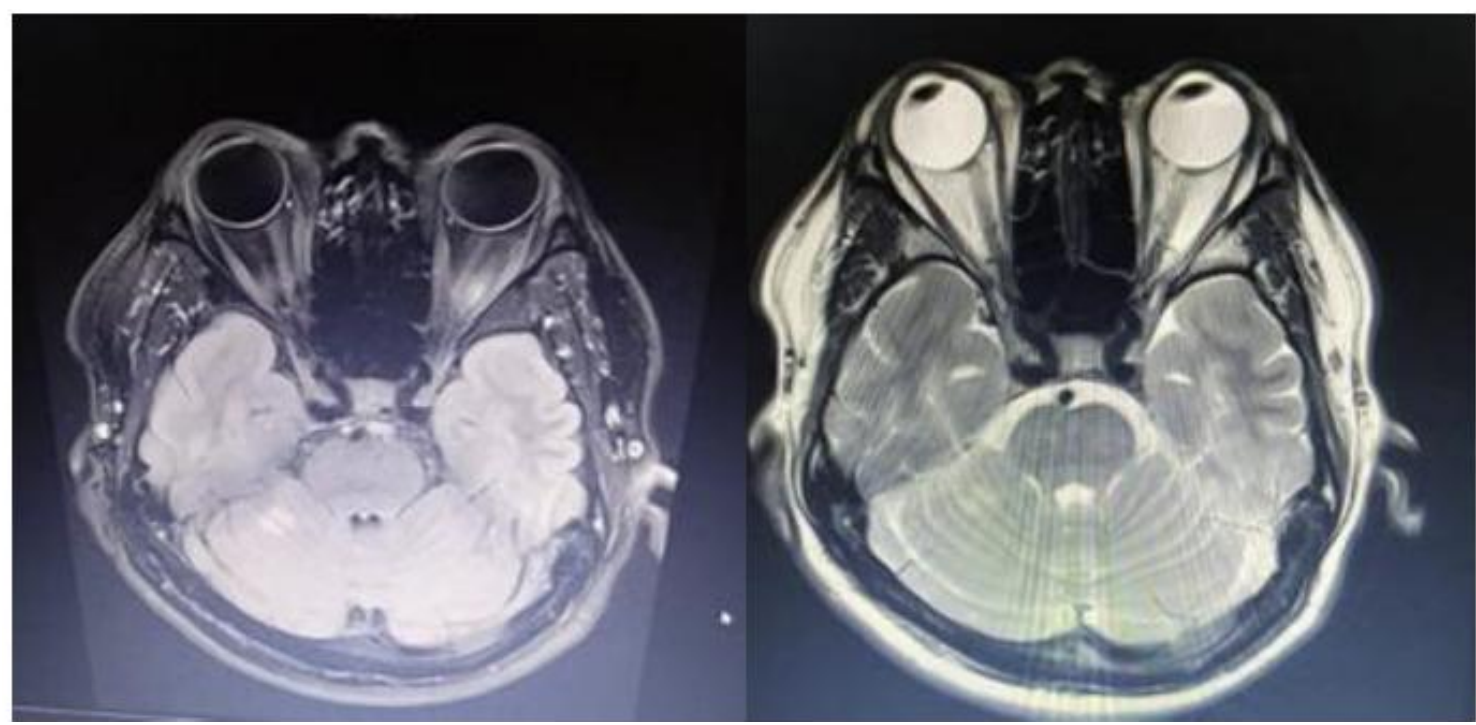

Figure 2. Magnetic Resonance Imaging (MRI) of the Head; Restricted diffusion on Diffuse weight Imaging (DWI), cerebellar hyperintense on T2 Fluid Attenuates Inversion Recovery FLAIR

A 41-year-old man suffered from acute-onset vertigo and dizziness about 5 hours before admission, which started when he started doing his morning routine. The patient described that the head is floating as if it is light and everything around him seemed to be moving. The symptoms were not prompted by changes in his head, and 
they did not improve with sleep. The patient stated that he would want to fall to his left side. The patient's hands and feet are unaffected. On the route to the hospital, the patient vomits, his head jerks, and he becomes uneasy. He denied any previous headaches, tingling, visual field abnormalities, double vision, swallowing difficulties, convulsions, or fever. The patient's previous medical history includes hypertension from 5 to 6 years ago, which was not routinely treated, and no previous stroke, tumor, or lump elsewhere, which the patient disputed. Traditional medicine was used previously, and no painkillers were used. Patient has been a 15-year smoker who smokes 5-10 cigarettes per day and does not drink alcohol. There are no relatives with hypertension, heart disease, diabetes mellitus, or stroke in the family history. Blood pressure was 210/150 $\mathrm{mmHg}$, pulse was 98 beats per minute, respiratory rate was $18-20 x /$ minute, temperature $36.6^{\circ}$ Celsius on general examination. Bidirectional horizontal nystagmus without hearing loss, excellent motor function, and normal exteroceptive and proprioceptive sensory were discovered on neurological examination. Both sides of the extremities have normal deep tendon reflexes, with no abnormal or primitive reflexes. Dysmetria on the left side, left dysdiadochokinesia, positive Romberg's test, and a fall to the left side were the cerebellar signs. The Head Impulse HIT test revealed a bidirectional horizontal nystagmus and no ocular deviation, which were both within normal ranges. Fasting glucose readings were $113 \mathrm{mg} / \mathrm{dl}$, postprandial blood sugar was $150 \mathrm{mg} / \mathrm{dl}$; urea was $15 \mathrm{mg} / \mathrm{dl}$; serum creatinine was $1.57 \mathrm{U} / \mathrm{L}$; albumin was $4.5 \mathrm{~g} / \mathrm{L}$; SGOT was $18 \mathrm{U} / \mathrm{L}$; SGPT was $16 \mathrm{U} / \mathrm{L}$; CRP was 9.4; WBC was 13,200/l; $\mathrm{Hb}$ was $15.2 \mathrm{~g} / \mathrm{dl}$; platelets were $425,000 / 1$. HbA1C levels 5.7 percent, lipid profile: cholesterol $245 \mathrm{mg} / \mathrm{dl}$, triglycerides $110 \mathrm{mg} / \mathrm{dl}$, HDL $42 \mathrm{mg} / \mathrm{dl}$, LDL $89 \mathrm{mg} / \mathrm{dl}$, electrolyte levels sodium $145 \mathrm{mmol} / \mathrm{L}$, potassium $4.0 \mathrm{mmol} / \mathrm{L}$, chloride $103 \mathrm{mmol} / \mathrm{L}, \mathrm{HbA} 1 \mathrm{C}$ values 5.7 percent. Cerebellar infarct, hypodense region in the cerebellum, can be seen on a CT scan of the head without contrast.

After the initial phase of the stroke, patients were given betahistine $24 \mathrm{mg}$ every 12 hours, Amlodipine $10 \mathrm{mg}$ every 24 hours, Domperidone 1 tablet every 12 hours or as required, Acetyl Salicylic Acid $100 \mathrm{mg}$ every 24 hours, Simvastatin $20 \mathrm{mg}$ every 24 hours, and Cawthorne-Cooksey exercise.

\section{DISCUSSION}

Acute vestibular syndrome (AVS) is characterized by acute onset of prolonged vertigo (days to weeks) with spontaneous nystagnation, postural instability and autonomic symptoms such as nausea or vomiting. $(1,4,5)$ Eleven percent of patients with cerebellar infarction showed clinically isolated vertigo and most (96\%) had medial branch PICA territory infarction, including nodules. (6) Cerebellar stroke will cause severe ataxia, visual disturbances, headache, and vertigo. However, about $10 \%$ of patients with cerebellar infarction experience only vertigo or nausea and vomiting. (7)

Pathophysiology of acute vestibular syndrome on cerebellum lesions is associated with vestibular pathway and vascularisation into the vestibular system. The cerebellum receives projections from the vestibular labyrinth, vestibular nerve, and vestibular nucleus in the brainstem and the projections back to the vestibular nucleus to control oculomotor and postural reflexes. The cerebellum is also involved in the visual suppression of ocular vestibular responses, including nystagmus caused by acute vestibular dysfunction. (8) The blood supply to the peripheral vestibular labyrinth, vestibular nerve, the vestibular nucleus in the brainstem and cerebellum originates from the vertebrobasilar artery system. $(8,9)$ The internal auditory arteries branch is a branch of the AICA's Anterior Inferior Cerebellar Artery, supplying the vestibular and cochlear labyrinths. Branches of the vertebral and basilar arteries supply the vestibular nuclei in the brainstem. $(6,8)$ The 
posterior and anterior cerebellar arteries provide blood supply to the inferior cerebellum and the flocculonodular lobe which are parts of the cerebellum that are closely related to the vestibular system. $(6,8)$

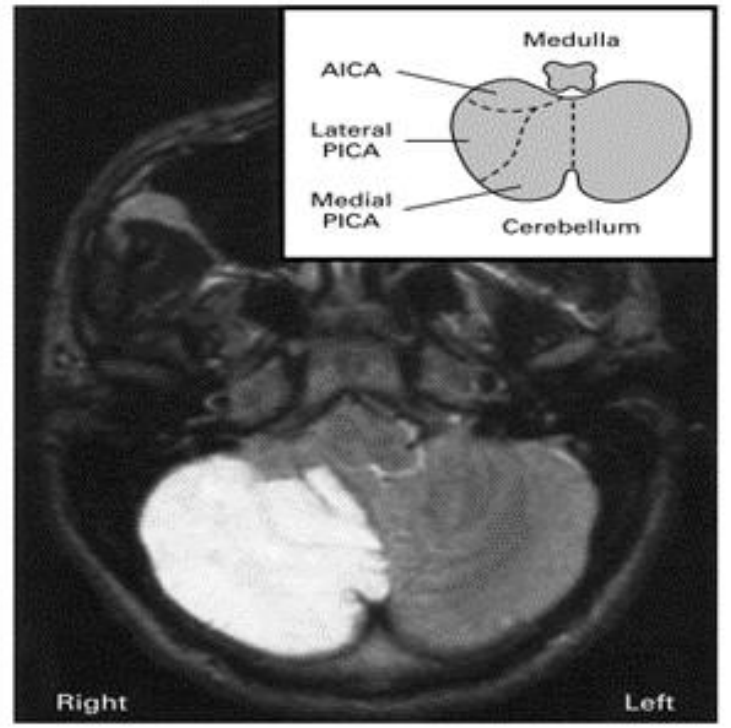

Figure 3: Magnetic Resonance Imaging MRI of right inferior cerebellar infarction. Pathophysiological vascularization illustration posterior inferior cerebellar artery (PICA) and anterior inferior cerebellar artery (AICA). (8)

The pathophysiology of acute vestibular syndrome also occur through involvement of the structures of the vestibular nucleus, root entry zone of cranial nerve VIII at the border of the pontomedula, dorsolateral or caudolateral rostral medulla, paramedian pons or mesencephalon tegmentum, and inferior cerebellar peduncle. (6) Cerebellar vestibular ocular reflex (VOR) function and associated vestibular abnormalities. Purkinje Cell of vestibulocerebellum have an inhibitory effect, usually in the ipsilateral vestibular nucleus. Damage of these Purkinje cells tend to increase the tonic activity of ipsilateral vestibular nucleus, causing spontaneous cerebellar nystagmus on the lesion side. However, patients with floccular lesions exhibit much stronger spontaneous nystagmus in response to abnormal head impulses, suggesting that the flocculus has a much more important role in controlling VOR than does the cerebellar tonsil. (10)
Diyan et al, 2020 and Kim Ah, 2012, described the most common causes of posterior circulation infarcts are posterior inferior cerebellar artery (PICA) and superior cerebellar artery (SCA) compared to the anterior inferior cerebellar artery (AICA). Patients with cerebellar infarction have clinical features of vertigo, nausea and vomiting, horizontal nystagmus, limb ataxia, unbalanced gait, and headache, may be in the occipital, frontal, or upper cervical region. Frequently, the clinical picture of cerebellar artery infarction results from infarction of the lateral medulla or pons, and does not involve the cerebellum. These clinical features include trigeminal and spinothalamic sensory deficits or Horner's syndrome. $(5,6)$ The patient we reported also presented with acute vertigo, head discomfort, such as floating sensation accompanied by a feeling of tumbling at the acute onset and may lead to acute-onset limb ataxia and associated vascular risk factors.

Also mentioned by Hotson and Baloh, 1998; The sudden onset of isolated vertigo lasting for minutes in a person with risk factors for stroke suggests an ischemic attack in the vertebrobasilar system or transient ischemia of the vestibular labyrinth. Transient ischemic attacks often last for less than 30 minutes. Isolated transient vertigo may precede a stroke in a branch of the vertebrobasilar artery that presents with similar symptoms over a span of weeks or months. (8) In acute vestibular syndrome, factors that lead to stroke should be considered, such as; age over 60 years, history of hypertension or diabetes mellitus, accompanying symptoms refer to the central nervous system, spontaneous and acute onset of symptoms for the first time. (11)

Bed side examination of acute vestibular syndrome is important to establish the diagnosis, even considered sensitive and specific for acute vestibular syndrome, it's hard to differentiate vascular vertigo isolated from peripheral vestibulopati acute. As described by Kim JS and Choi KD, 2013, indicates that bed side 
examination of oculomotor has three steps for hints: Head impulse test, nystagmus and Test of Skew Deviation consists of a head Impulse Test HIT, nystagmus, and the deviation of the eye is more sensitive to stroke while scheduling definitive imaging. $(1,2)$

If a patient shows any signs of central vestibular dysfunction such as vertical nystagmus in usual position, nystagmus due to head shaking movements, asymmetric oculomotor dysfunction, or postural instability that is severe enough to fall during a vertigo attack, the doctor can easily detect that the vertigo stems from central vestibular dysfunction. (5) An examination protocol that combines the Head Impulse test HIT with looking for nystagmus that changes direction in eccentric gaze or skew deviation is $100 \%$ sensitivity and $96 \%$ specificity for stroke identification, whereas diffusion weighted DWI Magnetic Resonance Imaging MRI can be missed in $12 \%$ of cases. $(5,12)$

Table 1. Characteristics of the clinical presentation of acute unilateral vestibulopathy, stroke in the posterior inferior cerebellar artery PICA and stroke in the anterior inferior cerebellar artery AICA (13)

\begin{tabular}{|l|l|l|l|}
\hline & Acute Unilateral Vestibulopathy & Stroke (PICA) & Stroke (AICA) \\
\hline Nystagmus & Spontaneous horizontal unidirectional & $\begin{array}{l}\text { Central form } \\
\text { nystagmus }\end{array}$ & Central form of nystagmus \\
\hline Qualitative Head Impuls Test & $\begin{array}{l}\text { Abnormal to one side, fast phase } \\
\text { nystagmus }\end{array}$ & Normal bilateral & $\begin{array}{l}\text { Abnormal on the side of the } \\
\text { lesion }\end{array}$ \\
\hline $\begin{array}{l}\text { Quantitative Head impuls Test } \\
\text { gain) }\end{array}$ & $\begin{array}{l}\text { Ipsilateral;0.2-0.4 } \\
\text { Contralateral: } 0.6-1.0\end{array}$ & $\begin{array}{l}\text { Ipsilateral;0.7-0.8 } \\
\text { Contralateral:0.7-0.8 }\end{array}$ & $\begin{array}{l}\text { Ipsilateral;0.3-0.4 } \\
\text { Contralateral: } 0.5-0.6\end{array}$ \\
\hline Skew deviation & - & There's a possibility & There's a possibility \\
\hline $\begin{array}{l}\text { Vascular Risk } \\
\text { Hearing disorders }\end{array}$ & Low Risk & High Risk & Often there \\
\hline
\end{tabular}

HINTs examination is useful to reduce misdiagnosis of stroke or acute vestibular syndrome and should be studied in head-to-head for the comparative costeffectiveness of the neuroimaging by MRI DWI. (13) The research of Kattah et al, 2009 and Choi et al, 2017 also proves that finding one of the 3 dangerous oculomotor signs (normal Head Impulse Test HIT or ni horizontal stagmus that changes direction in eccentric gaze or skew deviation) is more sensitive than the presence of other neurologic signs to identify if the acute vestibular syndrome is caused by stroke. $(14,15)$ The patient in this case report showed 2 out of 3 HINTS examination results that lead to stroke, namely normal on the Head Impulse Test (HIT) and the presence of bidirectional horizontal nystagmus, plus a deficit in the cerebellar system such as acute limb ataxia and cerebellar signs, dysmetria.

A computed tomography (CT) scan of the head is routinely done in individuals with acute vestibular dysfunction. CT is a fairly reliable diagnostic for cerebral bleeding; however, when conducted within
12 hours of the beginning of the event, the sensitivity of CT for ischemic stroke is just $39 \%$. With a sensitivity of 99 percent, Magnetic Resonance Imaging (MRI) with sequence Diffusion Weight Imaging (DWI) is significantly more accurate. Another study discovered that the sensitivity of MRI is reduced in minor lesions, stroke at the fossa posterior region, and if the MRI is conducted within 24 hours of the beginning of symptoms rather than within 2 to 4 hours. The DWI MRI procedure might be to blame for some of the decreased accuracy. (13) However, a recent meta-analysis indicated that, whereas only $6.8 \%$ of patients with ischemic stroke had negative DWI MRI findings, posterior circulation strokes had this outcome five times more frequently. Concurrent clinical evaluation and vascular imaging (CT or MR angiography to detect the relevant occlusion or stenosis) may be useful in determining a diagnosis. (9)

In most cases, only CT is performed, although in fact MRI is a better method for visualizing pathology in the posterior cranial fossa as demonstrated by the higher diagnostic yield of MRI in this and other 
studies. The use of CT as the only diagnostic investigation has several reasons, including; limited medical resources, unreasonable fears or concerns of patients (false sense) and prevention of exposure to ionizing radiation. It is important to be more selective when selecting patients for neuroradiological treatment, and in larger cases MRI can be used instead of CT because of the higher sensitivity for posterior fossa pathology. (3) Ideally, patients with acute dizziness require imaging. MRI is preferred over CT scan in the majority of cases. In certain cases, it is important to exclude bleeding prior to thrombolysis or to detect vertebral artery dissection using CT angiography. (4) The timing of MRI is also very important, because of the risk of false negatives in the first 48 hours. In some cases, it is necessary to perform a repeat MRI if the HINTS test results suggest a central process but the MRI results do not show any significance. (4) Imaging performed on our patient was a head-CT scan, which was performed at the time the patient was admitted to the emergency department, 5 hours of onset and found a suspected cerebellar infarction according to Fig. 1, then on the sixth day of treatment, the patient underwent MRI of the Head and was found to have infarction in the cerebellar region as per Fig. 2 with Magnetic Resonance Angiography (MRA) results within normal limits. These imaging results support the diagnosis of acute vestibular syndrome in this patient.

The treatment of acute vestibular syndrome is based on treating acute stroke; however, because this is linked with intravenous thrombolysis, it must be modified to the time and severity of the disease according to the National Institute of Health Stroke Scale (NIHSS) when the patient arrives. (16) About $4 \%$ of patients with dizziness or vertigo and clinical symptoms indicative of a posterior circulation stroke arrive to the emergency room, but most have past the 4.5-hour time window for intravenous thrombolysis therapy. If the score is low in acute vestibular patients, isolated vascular vertigo is not recommended for thrombolysis. Patients with an NIHSS score of more than four were evaluated for intravenous thrombolysis or urgent endovascular surgery. In the case of acute vestibular syndrome, conservative therapy will be effective. (16) Symptomatic therapy may be administered in the event of a labyrinth, cerebellum, or brainstem infarction. Exercise-based vestibular therapy is necessary as soon as feasible. There may be some improvement after therapy, but it is not usually complete. Patients with infarcts should be closely monitored to minimize brainstem compression caused by cerebellar edema. (17) The patient in our case report got symptomatic therapy for vertigo and dizziness in the form of Betahistine Hydrochloride $24 \mathrm{mg}$ every 12 hours, however thrombolysis could not be done because it had been more than 4.5 hours from the beginning when the patient arrived at the neurology department.

Prognosis for Acute vestibular syndrome will improve with time and will necessitate vestibular therapy. (13) Because of central structural damage, dizziness or vertigo might linger for months. Patients may also have discomfort in their heads or eyesight, which may seem unsteady, as well as spontaneous nystagmus and impairment to the central vestibular and cerebellar circuits. (17)

Antihistamines betahistine hydrochloride $24 \mathrm{mg}$ every 12 hours for 14 days, Acetyl Salicylic Acid $10 \mathrm{mg}$ every 24 hours, Amlodipine $10 \mathrm{mg}$ every 24 hours starting upon this fifth day after onset, Simvastatin $20 \mathrm{mg}$ every 24 hours were prescribed to our patient as symptomatic treatment. On the tenth day, the patient's symptoms improved, and his vertigo was significantly reduced by around 50-60\%, and his postural disturbances were reduced as well, allowing him to walk more steadily. The patient was then taught how to perform Cawthorne Cooksey vestibular therapy at home. 


\section{CONCLUSION}

Acute vestibular syndrome is closely related to the posterior circulation and can be caused by cerebellar infarction. Pathophysiology according to cerebral blood circulation disorders that occur. Clinical features of patients with cerebellar lesions may include vertigo, nausea and vomiting, horizontal nystagmus, limb ataxia or postural disturbances. The Bedside HINTS oculomotor examination is sensitive for acute vestibular stroke. Management Pharmacological therapy in the form of symptomatic and non-pharmacological in the form of vestibular rehabilitation. Investigations can be followed by $\mathrm{CT}$ or MRI of the head. The prognosis for acute vestibular syndrome is good.

\section{Acknowledgement: None}

\section{Conflict of Interest: None}

\section{Source of Funding: None}

\section{REFERENCES}

1. Kim SH, Kim HJ, Kim JS. Isolated vestibular syndromes due to brainstem and cerebellar lesions. J Neurol. 2017;264:63-9.

2. Choi KD, Lee H, Kim JS. Vertigo in brainstem and cerebellar strokes. Curr Opin Neurol. 2013;26(1):90-5.

3. Ljunggren M, Persson J, Salzer J. Dizziness and the Acute Vestibular Syndrome at the Emergency Department: A PopulationBased Descriptive Study. Eur Neurol. 2018;79(1-2):5-12.

4. Putra IBK, Adrian F. Dizziness dan Vertigo Dengan Keterkaitan Sistem Vertebrobasiler. Callosum Neurol. 2019;2(1):19-27.

5. Kim HA, Lee H. Recent advances in central acute vestibular syndrome of a vascular cause. J Neurol Sci [Internet]. 2012;321(12):17-22. Available from: http://dx.doi.org/10.1016/j.jns.2012.07.055

6. Diyan Anita Sari, Sri Sutarni IS. Stroke Iskemik Dengan Manifestasi Dizziness/Vertigo Terisolasi. Neurona. 2020; volume 37(2 Maret 2020):3-9.
7. Volgger V, Gürkov R. Acute vestibular syndrome in cerebellar stroke. HNO. 2017;65(07 march 2017):149-52.

8. John R. Hotson , M.D., And Robert W. Baloh MD. Acute Vestibular Syndrome. N Engl J Med. 1998; volume 339:9-14.

9. Banerjee G, Stone SP, Werring DJ. Posterior circulation ischaemic stroke. BMJ. 2018;361(April):1-7.

10. Kim SH, Park SH, Kim HJ, Kim JS. Isolated central vestibular syndrome. Ann N Y Acad Sci. 2015;1343(1):80-9.

11. Strupp M, Dlugaiczyk J, Ertl-Wagner BB, Rujescu D, Westhofen M, Dieterich M. Vestibular disorders: Diagnosis, new classification and treatment. Dtsch Arztebl Int. 2020;117(17):300-10.

12. Chen L, Lee W, Chambers BR, Dewey HM. Diagnostic accuracy of acute vestibular syndrome at the bedside in a stroke unit. $\mathrm{J}$ Neurol. 2011;258(5):855-61.

13. Kerber KA. Acute Vestibular Syndrome. Semin Neurol. 2020;40(1):059-66.

14. Kattah JC, Talkad A V., Wang DZ, Hsieh YH, Newman-Toker DE. HINTS to diagnose stroke in the acute vestibular syndrome: Three-step bedside oculomotor examination more sensitive than early MRI diffusion-weighted imaging. Stroke. 2009;40(11):3504-10.

15. Choi JY, Lee SH, Kim JS. Central vertigo. Curr Opin Neurol. 2018;31(1):81-9.

16. Machner B, Choi JH, Neumann A, Trillenberg P, Helmchen C. What guides decision-making on intravenous thrombolysis in acute vestibular syndrome and suspected ischemic stroke in the posterior circulation? J Neurol [Internet]. 2020;(0123456789). Available from: https://doi.org/10.1007/s00415-020-10134-9

17. Sri Sutarni,Rusdy Gazali Malueka AG. Bunga Rampai Vertigo. In: Wibowo S, editor. Yogyakarta: ugmpres.ugm.ac.id; 2018. p. 73-88.

How to cite this article: Lestari DT, Hidayati HB. Acute vestibular syndrome in cerebellar infarction: a case report. International Journal of Research and Review. 2021; 8(9): 29-35. DOI: https://doi.org/10.52403/ijrr.20210906 